\title{
Sensors, Medical Image and Signal Processing
}

\section{Findings from the Section on Sensor, Signal and Imaging Informatics}

\author{
R. Westphal, Managing Editor for the IMIA Yearbook Section on Sensor, Signal and Imaging \\ Informatics \\ Technical University of Braunschweig, Institute for Robotics and Process Control, Braunschweig, \\ Germany
}

\section{Introduction}

Besides of the classical fields of medical imaging informatics in diagnosis and therapy like outlined in [1], the scope of sensors, signal and imaging informatics (cp. [2]) comprises further recently emerging fields of applications. Meaningful examples are the support of disabled persons or the surveillance of persons with specific risk factors. The brain machine interface (BMI) community was increasing rapidly during the last two decades [3] and produced forward-looking applications ranging from the control of mouse cursors on a computer screen over the steering of wheelchairs [4] to the control of prostheses or robotic arms [5], which can support paralyzed persons during reaching and grasping tasks. Even though mature applications of BMI are already possible, there are still many open questions at the basics of the BMI technology (for example [6]).

Under the influence of aging societies, the relevance of telemedicine systems is increasing and guidelines for designing medical telecare services become important [7]. It could be shown, that the application of telemedicine systems can become a cost-effective solution in the treatment of elderly patients $[8,9]$. In this context, telemonitoring applications like presented by Istrate et al. [10] and body sensor networks [11,12] play an important role.
The paper selection for this section of the yearbook focused especially on applications with a possible short term relevance for patient care, which is in accordance to the practice of earlier yearbooks $[13,14,15,16]$.

\section{Best Paper Selection}

Ten international peer reviewed journals in the fields of medicine, signal and image processing and medical informatics were reviewed in order to find best papers for this year's section "Sensor, signal and imaging informatics". Additionally, a PubMed search with keywords according to this field was performed. Table 1 presents the selected papers. A brief content summary of the best papers can be found in the appendix of this report.

The first paper [17] presents a soft tissue and needle deflection model utilizing the precise location of needles in the patient's soft tissue during tasks like biopsies, brachytherapies, or anaesthesias. A sound surveillance telemonitoring system is present by Istrate et al. [10]. Two papers from the field of brain machine interfaces (BMI) have been selected. The paper of Kim et al. [5] presents a robotic system for supporting paralyzed persons during reaching and grasping tasks using a shared control concept, which integrates a brain controlled trajectory planning with reflex like reac- 
Table 1 Best paper selection of articles for the IMIA Yearbook of Medical Informatics 2007 in the section 'Sensor, Signal and Imaging Informatics'. The articles are listed in alphabetical order of the first author's surname.

\section{Section}

Sensor, Signal and Imaging Informatics

- DiMaio SP, Salcudean SE. Needle Steering and Motion Planning in Soft Tissues. IEEE Trans Biomed Eng 2005; 52(6): 965-74.

- Istrate D, Castelli E, Vacher M, Besacier L, Serignat JF. Information Extraction From Sound for Medical Telemonitoring. IEEE Trans Inf Technol Biomed 2006; 10(2): 264-74.

- Kim HK, Biggs SJ, Schloerb DW, Carmena JM, Lebedev MA, Nicolelis MAL, Srinivasan MA. Continuous Shared Control for Stabilizing Reaching and Grasping With Brain-Machine Interfaces. IEEE Trans Biomed Eng 2006; 53(6): 1164-73.

- Moore Jackson MM, Mason SG, Birch GE. Analyzing Trends in Brain Interface Technology: A Method to Compare Studies. Ann Biomed Eng 2006; 34(5): 859-78.

- Pärkkä J, Ermes M, Korpipää P, Mäntyjärvi J, Peltola J, Korhonen I. Activity Classification Using Realistic Data From Wearable Sensors. IEEE Trans Inf Technol Biomed 2006; 10(1): 119-28.

tions from sensors located at the gripper. The problem of objectively comparing studies in the diverse brain interface (BI) community is addressed by the paper Moore et al. [3]. They present a new methodology for comparing and distinguishing between BI studies. The fifth paper by Pärkkä et al. [18] presents a way to measure the activity of a person using body sensors, aiming at increasing the user's awareness of his daily activity level and therefore promoting a more active and healthy life style.

\section{Conclusions and Outlook}

Five excellent articles, representing the research in four different nations, are the result of the selection process for this yearbook section. The selection indicates, how advances in fields like patient modeling, brain machine interfaces, body sensors, and telemonitoring may positively affect future patient care. Up-to-date information about current and future issues of the IMIA Yearbook is available at http://www.schattauer.de/ index.php?id=1384.

\section{Acknowledgement}

I greatly acknowledge the support of Martina Hutter and of the reviewers in the selection process of the IMIA Yearbook and Prof. Haux and Kulikowski for giving me the opportunity to participate in selecting articles for this yearbook.

\section{References}

1. Lehmann TM, Meinzer HP, Tolxdorff T. Advances in biomedical image analysis - Past, present and future challenges. Methods Inf Med 2004; 43(4): 308-14.

2. Lehmann TM, Aach T, Witte H. Sensors, Signal and Image Informatics. Methods Inf Med - Supplement 2006, IMIA Yearbook: 57-67.

3. Moore Jackson MM, Mason SG, Birch GE. Analyzing Trends in Brain Interface Technology: A Method to Compare Studies. Ann Biomed Eng 2006; 34(5): 859-78

4. Millán J, Renkens F, Mouriño J, Gerstner W. Noninvasive Brain-Actuated Control of a Mobile Robot by Human EEG. IEEE Trans Biomed Eng 2004; 51(6): 1026-33.

5. Kim HK, Biggs SJ, Schloerb DW, Carmena JM, Lebedev MA, Nicolelis MAL, et al. Continuous Shared Control for Stabilizing Reaching and Grasping With Brain-Machine Interfaces. IEEE Trans Biomed Eng 2006; 53(6): 1164-73.

6. Gysels E, Renevey P, CelkaP. Fast Feature Selection to Compare Broadband with Narrowband Phase Synchronization in Brain-computer Interfaces. Methods Inf Med 2007; 46(2): 160-3.

7. Gortzis LG. Designing and Redesigning Medical Telecare Services - A Forces-oriented Model. Methods Inf Med 2007; 46(1): 27-35.

8. Bashshur RL. State-of-the-art telemedicine/ telehealth: ch. 1 telemedicine and health care. Telemed J e-Health 2002; 8(1): 5-12.

9. Jennett PA, Hall LA, Hailey D, Ohinmaa A, Anderson $\mathrm{C}$, Thomas R, et al. The socio-economic impact of telehealth: A systematic review. J Telemed Telecare 2003, 9(6): 311-20.

10. Istrate D, Castelli E, Vacher M, Besacier L, Serignat JF. Information Extraction From Sound for Medical Telemonitoring. IEEE Trans InfTechnol Biomed 2006; 10(2): 264-74
11. Lukowicz P, Kirstein T, Tröster G. Wearable Systems for Health Care Applications. Methods Inf Med 2004; 43: 232-8.

12. Bott OJ. Health Information Systems: Between Shared Care and Body Area Networks- Findings from the Section on Health Information Systems. Methods Inf Med - Supplement 2006, IMIA Yearbook: 53-6.

13. Dua S, Iyengar SS. Advances in Medical Signal Processing. Synopsis. IMIA Yearbook of Medical Informatics 2004

14. Pullan A. Biomedical Imaging. Synopsis. IMIA Yearbook of Medical Informatics 2004.

15. Barillot C. Medical Signal Processing and Biomedical Imaging. Synopsis. IMIA Yearbook of Medical Informatics 2005.

16. Westphal R, Winkelbach S. Sensors, Medical Image and Signal Processing - Findings from the Section on Sensors. Signal and Imaging Informatics. Methods Inf Med - Supplement 2006, IMIA Yearbook: 68-71.

17. DiMaio SP, Salcudean SE. Needle Steering and Motion Planning in Soft Tissues. IEEE Trans Biomed Eng 2005; 52(6): 965-74.

18. Pärkkä J,Ermes M, Korpipää P, Mäntyjärvi J, Peltola J, Korhonen I. Activity Classification Using Realistic Data From Wearable Sensors. IEEE Trans Inf Technol Biomed 2006; 10(1): 119-28.

19. Azar FS, Metaxas DN, Schnall MD. A finite element model of the breast for predicting mechanical deformations during biopsy procedures. in Proc. IEEE Workshop Mathematical Methods in Biomedical Image Analysis, 2000, pp. 38-45.

20. De Andres J, Reina MA, Lopez-Garcia A. Risks of regional anaesthesia: Role of equipment-Needle design, catheters. in Proc. 17th Annu. Eur. Soc. Regional Anaesthesia Congr., Sep. 1998, [Online]. Available: $\mathrm{http}: / \mathrm{www}$.esraeurope.org/andres14.htm.

21. Kohn LT, Corrigan JM, Donaldson MS. To Err Is Human: Building a Safer Health System. New York: Nat. Acad.; 2000

22. Hagendoorn H, Vuori I, Oja P. Guidelines for the development of national policies and strategies for promoting health through physical activity, The European Network for the Promotion of HealthEnhancing Physical Activity 2004; [Online]. Available: http://www.who.int/gb/ebwha/pdf files/ WHA57/A57 R17-en.pdf

23. Pate RR, Pratt M, Blair SN, Haskell WL, Macera CA, Bouchard C, et al. Physical activity and public health: a recommendation from the centers for Disease Control and Prevention and the American College of Sports Medicine. JAMA 1995, 273(5): 402-7.

\section{Correspondence to:}

Ralf Westphal

Technical University of Braunschweig

Insitiute for Robotics and Process Control

Muehlenpfordt Str. 23

D-38106 Braunschweig

Germany

Tel: +495313917463

Fax: +49531 3917445

E-mail: rwe@rob.cs.tu-bs.de 
Appendix: Content Summaries of Selected Best Papers, Section Sensor, Signal and Imaging Informatics*

\section{DiMaio SP, Salcudean SE \\ Needle Steering and Motion Planning in Soft Tissues}

IEEE Trans Biomed Eng 2005; 52(6): 965-74

The insertion of needles deep through the patient's soft tissue in order to precisely located a target, like required during biopsies, brachytherapy, or anaesthesia, is still a complicated task, as complex interactions between the needle and the deformable soft tissue might lead to conspicuous deflections of the planned trajectory. Errors in the target location or even injuries of surrounding structures may result $[19,20]$. Although the incidence of such complications is only in the order of a few percent [20], their consequences may be significant and even fatal [21]. This paper [17] presents a needle steering concept, which is based on numerical models incorporating needle deflections and soft tissue deformations. Path planning and obstacle avoidance in the soft tissue is achieved by combining these models with potential-field approaches. Trajectory planning and analysis methods like this could be applied in the future to support practitioners during needle insertion by means of robotic aids or navigation systems.

\section{Istrate D, Castelli E, Vacher M, Besacier L, Serignat JF \\ Information Extraction From Sound for Medical Telemonitoring}

\footnotetext{
* The complete papers can be accessed in the Yearbook's full electronic version, provided that permission has been granted by the copyright holder(s)
}

IEEE Trans Inf Technol Biomed 2006; 10(2): 264-74

Due to the actual growth of the aging population, the need for healthcare professionals and facilities is increasing. It could be shown, that the application of telemedicine systems can become a cost-effective solution in the treatment of elderly patients $[8,9]$. For telemonitoring, the application of video surveillance systems is broadly investigated. The authors of this paper [10] present a sound surveillance system, which might have the advantages of being more cost-effective and less intrusive to the patient's privacy, when compared to video surveillance systems. The first step of the proposed algorithm, extracts significant sounds from a continuous sound stream of a noisy environment, utilizing a discrete wavelet transform. In the second step, the detected sounds are classified using Gaussian mixture models. So classified sounds might also be fused with information from additional sensors to further improve the overall performance of telemonitoring systems.

\section{Kim HK, Biggs SJ, Schloerb DW, Carmena JM, Lebedev MA, Nicolelis MAL, Srinivasan MA}

\section{Continuous Shared Control for Stabilizing Reaching and Grasping With Brain-Machine Interfaces}

\section{IEEE Trans Biomed Eng 2006; 53(6): $1164-73$}

The combination of brain machine interfaces (BMI) with robotic actuators presents a promising and meaningful field of research with the prospect of enabling paralyzed individuals to manipulate their environment in the future. Kim et al. [5] present a robotic system for supporting paralyzed persons during reaching and grasping tasks. They use neuron recordings from a monkey to command a robot. A Con- tinuous Shared Control (CSC) architecture is used to achieve stability during the execution of these tasks. Using shared control, a brain-controlled trajectory is augmented by reflex-like reactions from sensors located in or near the robotic gripper. Therefore, the shortcomings of brain-machine interfaces, namely uncertainties about the intended trajectory coupled with low update rates of the command signals, can be compensated and the performance of such grasping tasks can be improved conspicuously over purely brain signal commanded executions.

\section{Moore Jackson MM, Mason SG, Birch GE. \\ Analyzing Trends in Brain Interface Technology: A Method to Compare Studies. Ann Biomed Eng 2006; 34(5): 859-78}

The research community of Brain Interfaces (BI) was expanding rapidly over the last two decades. Researchers from neuroscience, psychology, computer science, engineering, and medicine contribute to this interdisciplinary field. This diversity strengthens the field, and newer and more advanced technologies are reported constantly. However, this diversity also makes an objective comparison between studies and developments difficult. Moore et al. [3] proposed a methodology to directly compare studies of BI technologies. A representative set of 21 BI studies has been analyzed in this paper. Taxonomies like the one presented by Moore et al. can not only help in evaluating and objectively comparing BI studies by identifying their scopes and salient aspects. They furthermore allow for a characterization of overall trends in BI research or for the identification of research areas of inactivity.

\section{Pärkkä J, Ermes M, Korpipää P, Mäntyjärvi} J, Peltola J, Korhonen I 
Activity Classification Using Realistic Data From Wearable Sensors

IEEE Trans Inf Technol Biomed 2006; 10(1): 119-28

Physical inactivity is a health risk, which contributes to chronic diseases like cardiovascular disease or type 2 diabetes [22,23]. The recommended minimum daily physical activity of 30 minutes is not achieved by at least $60 \%$ of the world's population in developed as well as in developing countries according to the World Health Organization (WHO). By attaching wearable sensors to the user's body, the work of Pärkkä et al. aims at increasing the user's awareness of his daily activity level, which therefore promotes a more active and healthy lifestyle. In their paper [18], the authors discuss which sensors are applicable for this type of application. Furthermore, they present and evaluate different classification algorithms based on custom decision trees, automatically generated decision trees, and artificial neural networks for distinguishing between user activity and inactivity. 